six grains ; Mr. Lyster ${ }^{3}$ of Coleshill also described a case where recovery followed after a patient had probably taken two grains and a half of the alkaloid ; and Mr. Fegen ${ }^{4}$ described the symptoms in a fatal case where it was supposed that from two to three grains of the poison had been taken. Dr. Bingham ${ }^{5}$ of Alfreton was also engaged with a fatal case of poisoning from this drug. Dr. Wyman's suggestion with regard to the continued administration of chloroform appears a practical one. With regard to some of the antidotes which have been more recently recommended, Anrep ${ }^{6}$ thinks urethan superior to chloral hydrate and advises the administration of one to one and a half drachms when a poisonous dose of strychnine has been taken. Dr. C. Sanquirico recommends intravenous injections of a considerable quantity of an 8 per cent. soda solution, which will produce an actire diuresis and assist in eliminating the poison. J. S. Horsley in a case of strychnine poisoning injected apomorphine in doses of from one-fifteenth to one-tenth of a grain subcutaneously and "completely subdued the convulsions and eventually successfully antagonised the excitant alkaloid."

At 3 A. M. on April 12th, 1892, two women were brought to the hospital said to be suffering from the effects of poison through eating tinned salmon. It appeared that they had both dined off tinned salmon at 7 P. M. on the evening of the 11 th and supped off the same substance at $2 \mathrm{~A}$.M. on the 12th. But one of the women, E. S-, also stated that a man who left their house just before their supper at 2 A.M. had given them each "three long narrow pills" (capsules). The exact time at which the unfortunate women swallowed the pills could not be ascertained. At $2.30 \mathrm{~A}$. M. their screams aroused the landlord, and a policeman who was called in brought them to the hospital, having first given them each a mustard-and-water emetic, but without the production of vomiting. One of the women, A. M- - died on the way to the hospital. The other, E. S-, was alive and when first seen was sitting in a chair apparently quite well. Within a minute or two she had a convulsive attack in which, owing to the straightening of her back, she slipped off the chair on to the floor. An emetic (40 gr. of sulphate of zinc) was given but without effect. The stomach was then washed out several times with tepid water and half a tumblerful of thick charcoal-and-water given her to drink. During this time the jutient was having convulsive attacks every four or five minutes. The attacks were of the following character: The vatient suddenly became quite stiff, the back rigid and arched, with rigid flexure of arms and rigid extension of legs and feet. The rigidity was very marked during the greater portion of the attacks, which usually lasted from one minute to a minute and a half. But towards the end of the attacks, sometimes from the beginning, there were spasmodic movements of leos, arms and back. The pupils were equal, semi-dilated and reacted to light. The pulse was even but rather accelerated. Respiration seemed to stop during the attacks. The patient recovered from these fits almost as quickly as she entered them and in the intervals between them was perfectly conscious and rational, only complaining of a little stiffness of the legs and dryness of the throat. The attacks came on at fairly regular intervals, but whenever she received any external stimulus-such as change of position, the attempt to drink or even, on one or two occasions, the sound of pouring fluid into a glass-she had a fresh attack. The attacks were frequently preceded by a loud cry. As the fits did not abate in frequency or sererity chloroform was administered at about $415 \mathrm{~A}$. Mr. and while under its influence the patient was removed to Christian ward. While being put to bed the effect of the chloroform partially passed off and the patient had another attack, in which - he became greatly cyanosed and respiration was suspended. It was only after some minutes of artificial respiration that she again began to breathe. Chloroform was then again administered, and the patient was kept deeply under it influence until 730 A.M., during the whole time of it a tministration breathing quietly and maintaining a firm, steady pulse. At 7.30 it was discontinued under a false hope of security, and as its effects began to wear off the patient had three more convulsive attacks, in the last of which she died, although artificial respiration and cbloroform were again resorted to. Death took place with extreme cyanosis at 7.45 A.M., about five hours and a kalf after swallowing the pills. The u"ine, which had been retained since admission, was drawn off by catheter at 7 A.M., and was found

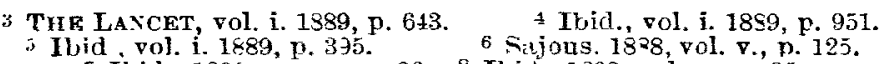

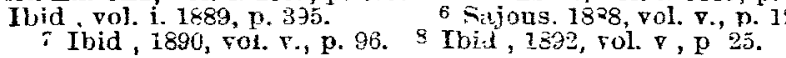

to contain a trace of albumen and a deposit of urates. It was afterwards analysed by Mr. White, the hospital pharmaceutist, who discovered in it one-twelfth of a grain of strychnine.

The post-mortem examination showed no sign of any organic disease in either case. In both cases the blood was very dark and fluid. In the case of E. S- the right side of the heart was distended with soft, black clot and the lungs were full of blood and were cedematous. In the case of A. M- both sides of the heart were empty, but the lungs were in the same condition as those of E. S-The stomachs, with their contents, a portion of liver and one kidney from each case were removed and forwarded to Dr. Stevenson, Government analyst, for examination. He reported the results of his analysis as follows :
E. S. - Vomit contained
Stomach and conte
Liver and kidney
$1 \cdot 46$
$1 \cdot 60$
1.60
0.20
$\begin{array}{llllll}\text { Total } & \ldots & \ldots & \ldots & \ldots & 326\end{array}$
A. M. - Stomach and contents $\ldots 6 \quad 639$
$\begin{array}{llll}\text { Liver and kidney } & \ldots & \ldots & 0.40\end{array}$
.40
$\begin{array}{llllll}\text { Total } & \ldots & \ldots & \ldots & \ldots & 6 \cdot 79\end{array}$

Remarks by Dr. WrMAN. - The symptoms as above detailed in the case of $\mathbf{E}$. S- coupled with the fact that $A . M-$ had already died from the effects of presumably the same poison, were so characteristic as to be unmistakable. As regards the treatment it may be said that as long as $\mathbf{E}$. $\mathbf{S}-$ remained under the influence of chloroform she appeared to be in no danger, at any rate from the effects of the poison. And it is reasonable to suppose that if the administration had been continued until the poison had had time to be eliminated from the system by the urine the patient might have recovered. That the administration would have had to have been continued for some twelve hours or more seems probable from the fact that only one-twelfth of a grain was so got rid of during the five hours she was under treatment. From this, then, it would seem that if the chloroform treatment is used at all it must be continued until all trace of the poison has disappeared. This can only be determined by watching very carefully for any sign of twitching of muscles, especially of those about the eye and mouth, during the period immediately succeeding the cessation of the administration of chloroform. If the slightest twitching is observed chloroform should be again administered. Unfortunately, the process of separating the strychnine from the urine is rather a lengthy one in point of time and therefore not likely to be of much value in this respect. Charcoal readily absorbs strychnine and other vegetable alkaloids and is therefore a useful remedy. It should be given in large quantities, allowed to remain in the stomach for ten minutes or a quarter of an hour and then washed out with the stomach pump, for if ailowed to remain and pass on into the intestines the strychnine will again be given off and carried into the circulation. The process should be repeated two or three times. Another point of great importance from a legal point of view which arose in this case is the question, When, with is view of obtaining a dying declaration, is the medical attendant justified in telling the patient that he is dying? Any description of the murderer or other statement concerning the crime cannot be brought as evidence against a prisoner unless it can be proved that the victim at the time of giving it believed that he was actually dying and would not live many hours. In this case I did not feel myself justified in telling the patient that she was dying and there fore her statements were not allowed as evidence at the trial It must be remembered that a living witness is better than a dead one, and anything tending to add to the risks of death in a doubtful case should be avoided. If, on the other hand, there can be no doubt that a fatal result is imminent, I think it is the medical attendant's duty to tell the patient plainly what his condition is, and so possibly assist justice and protect other people from a similar fate.

\section{TORBAY HOSPITAL, TORQUAY.}

\section{A CASE OF ACUTE MYELITIS ; COMPLETE RECOVERY.}

\section{(Under the care of Dr. Pow kll..)}

As Mr. Watson, the house surgeon, to whom we are indebted for the notes, observes, complete recovery from acute 
myelitis is rare." Dr. Wilks in his "Lectures on Diseases of the Nervous System" only quotes two cases and Dr. Pye-Smith mentions a case in "Fagge's Medicine." The cause in this case was most likely exposure to wet and cold and most probably the former had more to do with it than the latter. The suddenness of the attack was well marked, and retention of urine was one of the earliest symptoms. The patient improved gradually, but his improvement was seen from the day of his admission, so that it is somewhat improbable that the iodide of potassium had any beneficial effect, as this was not prescribed until two days after his admission to hospital. The absence of bedsores was in the patient's favour and every care was taken in preventing them, the patient being put on a water-bed and the skin of the back, buttocks and heels daily washed with methylated spirit and dusted with zinc and starch powder. One half of the spinal cord appears to have been more involved than the other half, as the paralysis was always more marked on the right side.

S. G-, aged forty-three, a trawler, was admitted on July 28th, 1891, complaining of paralysis of the lower extremities, slight pain in the lumbar region and retention of urine. The patient was a strongly built, dark-haired man about six feet in height. He gave the following history. A week before the commencement of his present illness he was cutting grass with a pair of shears and had to sit and lie about on the wet ground. He continued this occupation for a week. On July 18th he noticed that his feet felt "numb and dead" and in the afternoon he could not pass his urine and was obliged to send for his doctor and have it drawn off. The feeling of numbness extended from the lower part of the abdomen and down the thighs and legs. On the 21st his legs felt worse and he could not walk; the right leg was worse than the left. At the beginning of his illness he suffered from pain in the back; this was more marked on trying to sit up in bed. Fifteen years ago he had gonorrhoea, but had never had syphilis. He had been strong and healthy up to the time of his present illness. The heart and lungs were normal. Temperature $99^{\circ}$; pulse 68 , full, regular and not easily compressible. The legs and thighs were well developed, but the muscles felt distinctly flabby. $\mathrm{He}$ had complete paralysis of the right leg, but he could move the left leg very slightly. There was no plantar or cremasteric reflex on either side. Both patellar reflexes were increased, but more so on the right than on the left side. Ankle-clonus was well marked on both sides. Sensation was altered on the plantar surface of the feet; he conld not feel the prick of a pin, but stated that he felt something touching him. This modified sensation was also present on the legs and thighs, but was more marked on the plantar surface of the feet. The catheter was used to draw off urine, and as the urine was alkaline and ammoniacal his bladder was washed out twice a day with hot boracic lotion. His bowels were constipated, but before the illness he had always been regular. He was given a light diet, and a mixture of iodide of potassium ( $5 \mathrm{gr}$.) with carbonate of ammonia and gentian was ordered. This mixture was continued for three weeks. He had then gained more power in the legs, the right leg was still worse than the left and the muscles felt softer. His bowels were very troublesomeand only acted after an enema. He continued to improve and on Oct. 8th the following note was made. The patient's gait has much improved, he can now walk long distances without fatigue, but in walking he seems to bring his feet rather too suddenly down, and sometimes he totters slightly. The muscles of both legs feel much firmer. The right knee-jerk is still much increased and the left slightly, there is no ankle clonus. He passes urine about every five hours during the day and gets up once or twice in the night to pass it. He has been taking four minims of liquor strychniæ three times a day since Aug. 19th.

The patient left the hospital on Oct. 13th, and has been under observation since. He has been in his usual health for some months. He can walk long distances without fatigue and has resumed his occupation as a trawler. The muscles of both legs have completely regained their normal tone, his gait is quite natural and he has no increase of knee-jerks. The urine is acid and he has no trouble in passing it. His general health is now very good.

I See also Bristowe : Theory and Practice of Medicine.

The National Leprosy Fund.-It is understood that a few advance copies of the report of the Leprosy Commissioners may be expected to arrive in Fngland in the course of the present month.

\section{aftlobical Sorritics.}

\section{ROYAL MEDICAL AND CHIRURGICAL SOCIETY.}

\section{Effects of Todides on Arterial Tension and Excretion of Urates.}

AN ordinary meeting of this Society was held on Jan. 10th, Dr. George Harley, Vice-President, in the chair.

A telegram from the President was read announcing his inability to be present and asking the Society to welcome Dr. Mirza Ali, chief physician to the Shah and Professor of Medicine in the University of Teheran, who was on a visit to this country. He was accompanied by Hussein Kuli Khan, Secretary of the Persian Legation, and he presented to the library of the Society copies of his medical works in Persian and French.

Dr. A. HAIG read a paper on the Effects of the Iodides on Arterial Tension and the Excretion of Urates. He referred to an article of his in vol. lxxi. of the Transactions on drugs which diminished the excretion of urates. The list of substances which had this action had now been greatly enlarged and he believed that the iodides must be added to it. He also believed that the action of these substances on the solubility and excretion of urates would explain a large part of their value and utility in medicine and surgery, just as he had previously said elsewhere with regard to opium and mercury, which acted in the same way. He was at first misled by his results with the iodides, and was further hindered by their affecting the process which he had used for the estimation of uric acid (Haycraft's). These difficulties were, however, got over, and with greater knowledge and experience of the working of certain laws which govern the excretion of urates and of water he now believed that it was possible to speak more decidedly as to the action of iodides. One of these laws was that first formulated by the writer at the beginning of $1889^{1}$ : "that, cateris paribus, arterial tension varied with the uric acid that was circulating in the blood." Another was that from day to day and from hour to hour in physiological conditions the urinary water varied inversely as the uric acid was excreted along with it. Another was that in physiological conditions the excretion of urates in the urine varied inversely as the acidity of the urine. And another, that the amount of urate in the urine was relatively to the urea, to a certain extent an index of the amount of urate passing through the blood. From these it followed that arterial tension varied with the amount of uric acid that was being excreted in the urine. But arterial tension meant contracted arterioles, and contracted arterioles meant that water had difficulty in passing the kidneys, as was shown to be the case in the parallel action of digitalis and other drugs which contracted the arterioles, and this was the reason why the urinary water varied inversely as the uric acid. The diuretic action of iodides was well known, and the writer showed four figures which demonstrated that at the time an iodide was causing diuresis it was also causing a diminished excretion of urate and that the one thing was related to the other as cause and effect. The figures also showed well the inverse relation of urates and water in excretion; also that under the influence of iodides the excretion of urate ceased for a time to bear its usual inverse relation to acidity. But the writer pointed out that some twenty drngs, or rather groups of drugs, all diminished the excretion of uric acid in the urine, and at the time they did this they produced also relaxed arterioles, lowered arterial tension and diuresis. Then iodides could beclassed along with these drugs, and as the latter could further be broken up into three groups according to the way in which they produced the diminished excretion of uric acid it might be possible to say which of the groups the iodides most resembled in their mode of action. He pointed out how this action of iodides on the solubiiity of urates, and so on the contraction of arterioles, enabled us to explain all their most important effects in physiology and pathology, just as he had previously pointed out in the case of opium and mercury. Lastly, he referred to his previous writings or uric acid as a cause of high arterial tension and suggested that there was no possible explanation of the parallel action of all these drugs except that which he had given-namely, that urates contracted the arterioles all over the body and

1 Brit. Med. Jour, vol. i. p. 281. 